\title{
Investigation of association of microsomal TAG transfer protein gene MTTP-164 T>C promoter polymorphism with metabolic syndrome variables
}

\author{
A. AlSaleh, J. S. Y. Lui, S. O'Dell and T. Sanders \\ Nutritional Sciences Division, King's College London, Franklin-Wilkins Building, 150 Stamford Street, London, SE1 9NH
}

The microsomal TAG transfer protein (MTTP) is required for the assembly and secretion of apoB-containing lipoproteins from the intestine and liver ${ }^{(1)}$. Associations have been found between single nucleotide polymorphisms (SNP) in the MTTP gene and LDLcholesterol (LDL-C) and related traits of the metabolic syndrome. The MTTP gene promoter SNP T-164C (rs1800804) C allele has been shown to be associated with higher transcriptional activity in gene-reporter assays ${ }^{(2)}$.

Genotyping of T-164C was undertaken in 466 healthy male and female participants of the RISCK Study, a randomised parallel controlled trial that aims to test the impact of specific dietary changes on cardiovascular risk factors associated with the metabolic syndrome. The association between genotype and mean quantitative metabolic phenotypes, LDL-C, HDL-cholesterol (HDL-C), TAG, total cholesterol, apoB, NEFA and insulin sensitivity was tested. Baseline measurements were taken after following a reference diet (38\% fat, $18 \%$ SFA and $12 \%$ MUFA) for 1 month.

The numbers of each genotype were in Hardy-Weinberg proportions as follows: T/T 226, T/C 179, C/C 37; C allele frequency 0.29. No associations with mean quantitative metabolic phenotypes were found after adjustment for age, gender and BMI. A significant association was found with HDL-C in males, in whom the presence of the variant $\mathrm{C}$ allele was associated with lower fasting serum HDL-C $(P=0.013$; Figure). No significant variation was found in fasting serum LDL-C in relation to genotype.

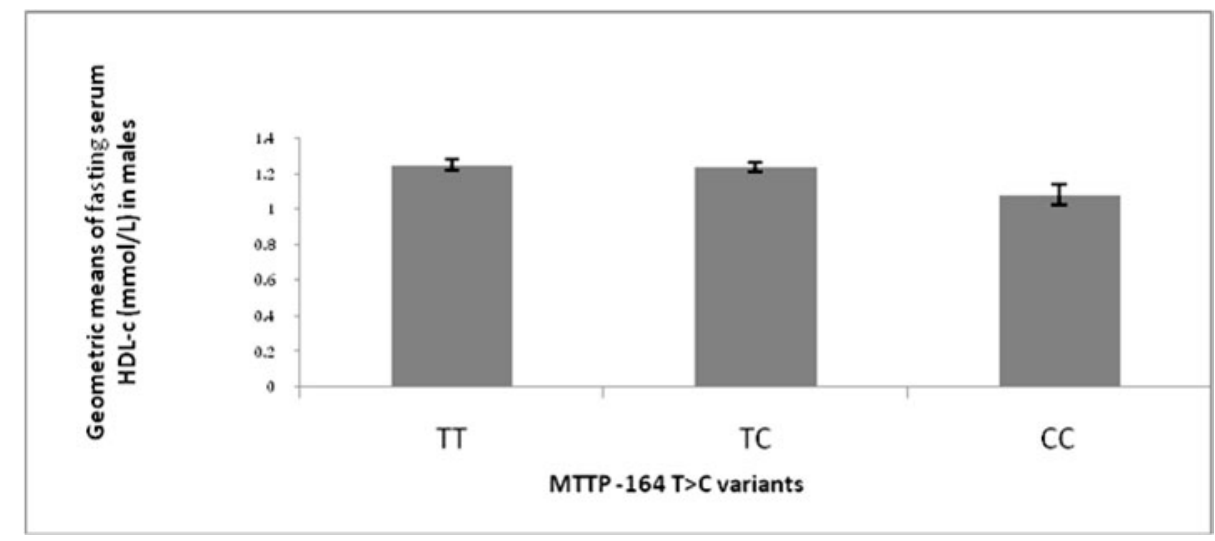

Figure. Baseline HDL-C concentrations according to MTTP $T-164 C$ variants in males. Values are means with their standard errors represented by vertical bars.

These findings suggest that the genetic variability at the MTTP $T-164 C$ locus may account for small inter-individual variation in fasting HDL-C concentration in obese and overweight males. Males homozygous for the $\mathrm{C}$ allele had significantly lower serum HDL-C compared with other genotypes, which may modulate risk of the metabolic syndrome.

1. Rubin D, Schneider-Muntau A, Klapper M et al. (2008) Human Mutat 29, 123-129.

2. Ledmyr H, Karpe F, Lundahl B et al. (2002) J Lipid Res 43, 51-58. 